\title{
Top-Dimensional Group of the Basic Intersection Cohomology for Singular Riemannian Foliations
}

\author{
by
José Ignacio ROYO PRIETO, Martintxo SARALEGI-ARANGUREN and Robert WOLAK

Presented by Czestaw BESSAGA

\begin{abstract}
Summary. It is known that, for a regular riemannian foliation on a compact manifold, the properties of its basic cohomology (non-vanishing of the top-dimensional group and Poincaré duality) and the tautness of the foliation are closely related. If we consider singular riemannian foliations, there is little or no relation between these properties.

We present an example of a singular isometric flow for which the top-dimensional basic cohomology group is non-trivial, but the basic cohomology does not satisfy the Poincare Duality. However, we recover the Poincaré Duality in the basic intersection cohomology.

It is not accidental that the top-dimensional basic intersection cohomology groups of the example are isomorphic to either 0 or $\mathbb{R}$. We prove that this holds for any singular riemannian foliation of a compact connected manifold. As a corollary, we show that the tautness of the regular stratum of the singular riemannian foliation can be detected by the basic intersection cohomology.
\end{abstract}

For regular riemannian foliations on compact connected manifolds there is a very close relation between tautness (the existence of a bundle-like metric for which all leaves are minimal submanifolds) and the properties of the basic cohomology. In fact, for a regular riemannian foliation $\mathcal{F}$ of codimension $n$ on a compact connected manifold $M$, under some assumptions of orientability

2000 Mathematics Subject Classification: 53C12, 53R30, 58A35, 55N30.

Key words and phrases: singular riemannian foliation, basic intersection cohomology, basic cohomology, taut foliation.

Research of J. I. Royo Prieto partially supported by the UPV-EHU grant 127.310E-14790/2002, by a PostGrant from the Gobierno Vasco-Eusko Jaurlaritza and by the $\mathrm{MCyT}$ of the Spanish Government.

Research of M. Saralegi-Aranguren partially supported by the UPV-EHU grant 127.310-E-14790/2002.

Research of R. Wolak partially supported by the KBN grant 5 PO3A 02125. 
the following conditions are equivalent (cf. $[3,4,9,10,24])$ :

(i) $\mathcal{F}$ is taut.

(ii) $H^{n}(M / \mathcal{F}) \neq 0$.

(iii) $H^{n}(M / \mathcal{F})=\mathbb{R}$.

(iv) $H^{*}(M / \mathcal{F})$ satisfies the Poincaré Duality $(\mathrm{PD})$.

Although the basic cohomology of a singular riemannian foliation (SRF for short) on a compact manifold is finite-dimensional (cf. [25]), the relation between the above conditions is not straightforwardly exportable to the singular framework. As pointed out in [11], the singular nature of a SRF on a compact manifold prevents any global metric on it from making all the leaves minimal (see also [17]).

The example presented in this paper shows that if, for flows, we replace "taut" by "isometric" - these two properties are equivalent for regular riemannian flows (see [15]) - we cannot recover PD. To recover it we have to adapt the basic cohomology to the stratification defined by the SRF. For that purpose, we have defined the "basic intersection cohomology" (BIC for short) for SRFs (see $[18,19])$. The calculations for the example show that its BIC satisfies the Poincaré Duality. It has been proven in [16] that so does the BIC of any singular riemannian flow (isometric or not).

The main part of this note is dedicated to the proof of the equivalence of conditions (i)-(iii) for SRFs on compact connected manifolds for the BIC. We also prove that the top-dimensional BIC groups are isomorphic to 0 or $\mathbb{R}$.

The authors would like to thank the referee for many useful comments which helped improve the paper.

In what follows, $M$ is a connected, second countable, Hausdorff, smooth $\left(C^{\infty}\right)$ manifold of dimension $m$ without boundary. All the maps considered are smooth unless otherwise indicated. If $\mathcal{F}$ is a foliation on $M$ and $V$ is a saturated submanifold of $M$ we shall write $(V, \mathcal{F})$ for the induced foliated manifold and $\mathcal{F}_{V}$ for the induced foliation.

1. Singular riemannian foliations $\left({ }^{1}\right)$. We are going to work in the framework of the singular riemannian foliations introduced by Molino.

1.1. The SRF. A singular riemannian foliation (SRF for short) on a manifold $M$ is a partition $\mathcal{F}$ of $M$ into connected immersed submanifolds, called leaves, with the following properties:

I. The module of smooth vector fields tangent to the leaves is transitive on each leaf.

$\left({ }^{1}\right)$ For notions relating to riemannian foliations we refer the reader to [13, 24] and for singular riemannian foliations to $[13,12,14,1]$. 
II. There exists a riemannian metric $\mu$ on $M$, called an adapted metric, such that each geodesic that is perpendicular to a leaf at one point remains perpendicular to every leaf it meets.

The first condition implies that $(M, \mathcal{F})$ is a singular foliation in the sense of [23] and [22]. Notice that the restriction of $\mathcal{F}$ to a saturated open subset produces a $\mathrm{SRF}$.

In the next two subsections we recall some basic properties of SRFs which can be found in $[13,1]$ or are easy corollaries of the properties proved there.

1.2. Stratifications. Classifying the points of $M$ according to the dimension of leaves yields a stratification $\mathrm{S}_{\mathcal{F}}$ of $M$ into smooth embedded submanifolds. The restriction of $\mathcal{F}$ to a stratum $S$ is a regular foliation $\mathcal{F}_{S}$. The strata are ordered by: $S_{1} \preceq S_{2} \Leftrightarrow S_{1} \subset \bar{S}_{2}$.

There are several types of strata. The minimal (resp. maximal) strata are the closed strata (resp. open strata). The open strata are called regular and the others singular. We denote by $\boldsymbol{S}_{\mathcal{F}}^{\sigma}$ the family of singular strata. In the case of SRFs, the singular strata are of codimension greater than 1, so there is just one regular stratum $R$ if the manifold is connected (cf. [13]). The dimension of the foliation $\mathcal{F}$ is the dimension of the biggest leaves of $\mathcal{F}$, that is, $\operatorname{dim} \mathcal{F}=\operatorname{dim} \mathcal{F}_{R}$. The union of the singular strata is the singular part $\Sigma=M \backslash R$.

A stratum $S$ is a boundary stratum if there exists a stratum $S^{\prime}$ with $S \preceq S^{\prime}$ and $\operatorname{codim}_{M} \mathcal{F}=\operatorname{codim}_{S^{\prime}} \mathcal{F}_{S^{\prime}}-1$. This terminology can be explained by the following example. Take $M=\mathbb{S}^{4}$ and $\mathcal{F}$ given by the orbits of the $\mathbb{T}^{2}$-action $(u, v) \cdot\left(z_{1}, z_{2}, t\right)=\left(u \cdot z_{1}, v \cdot z_{2}, t\right)$, where $\mathbb{S}^{4}=\left\{\left(z_{1}, z_{2}, t\right) \in \mathbb{C}^{2} \times \mathbb{R} \mid\right.$ $\left.\left|z_{1}\right|^{2}+\left|z_{2}\right|^{2}+t^{2}=1\right\}$. Here, the north pole $(0,0,1)$, the south pole $(0,0,-1)$ and the cylinders $\left\{z_{1} \neq 0\right\},\left\{z_{2} \neq 0\right\}$ are boundary strata and we have $M / \mathcal{F}=\mathbb{D}=\left\{(x, y) \in \mathbb{R}^{2} \mid x^{2}+y^{2} \leq 1\right\}$. The boundary $\partial(M / \mathcal{F})$ is given by $\mathbb{S}^{1}$, the union of the quotient set of the boundary strata. In fact, the link of the maximal boundary strata is a sphere with the one leaf foliation (see for example [18] for the notion of a link).

The depth of $\boldsymbol{S}_{\mathcal{F}}$, written depth $\boldsymbol{S}_{\mathcal{F}}$, is defined to be the largest $i$ for which there exists a chain of strata $S_{0} \prec S_{1} \prec \cdots \prec S_{i}$. So, depth $S_{\mathcal{F}}=0$ if and only if the foliation $\mathcal{F}$ is regular.

1.3. Tubular neighborhood. Any stratum $S \in \mathrm{S}_{\mathcal{F}}$ is a proper submanifold of the riemannian manifold $(M, \mathcal{F}, \mu)$, so it has a tubular neighborhood $\left(T_{S}, \tau_{S}, S\right)$. Recall that associated with this neighborhood are the following smooth maps:

- The radius map $\varrho_{S}: T_{S} \rightarrow[0,1[$ defined fiberwise: $z \mapsto|z|$. Each $t \neq 0$ is a regular value of $\varrho_{S}$. The pre-image $\varrho_{S}^{-1}(0)$ is $S$. 
- The contraction $H_{S}: T_{S} \times[0,1] \rightarrow T_{S}$ defined fiberwise: $(z, r) \mapsto r \cdot z$. The restriction $\left(H_{S}\right)_{t}: T_{S} \rightarrow T_{S}$ is an embedding for each $t \neq 0$ and $\left(H_{S}\right)_{0} \equiv \tau_{S}$.

These two maps satisfy $\varrho_{S}(r \cdot u)=r \varrho_{S}(u)$. The tubular neighborhood can be chosen to have the following two important properties:

(a) Each $\left(\varrho_{S}^{-1}(t), \mathcal{F}\right)$ is a $\mathrm{SRF}$.

(b) Each $\left(H_{S}\right)_{t}:\left(T_{S}, \mathcal{F}\right) \rightarrow\left(T_{S}, \mathcal{F}\right)$ is a foliated map.

We shall say that $\left(T_{S}, \tau_{S}, S\right)$ is a foliated tubular neighborhood of $S$. The existence of foliated tubular neighborhoods follows from the homothetic transformation lemma of [13].

The hypersurface $D_{S}=\varrho_{S}^{-1}(1 / 2)$ is the core of the tubular neighborhood. We have the inequality depth $S_{\mathcal{F}_{D_{S}}}<\operatorname{depth} S_{\mathcal{F}_{T_{S}}}$.

2. Basic intersection cohomology $\left({ }^{2}\right)$. Goresky and MacPherson introduced the intersection cohomology for the study of singular manifolds. This cohomology generalizes the usual de Rham cohomology for manifolds and has similar properties. Following the same principle, the basic intersection cohomology has been introduced for the study of SRFs, generalizing the basic cohomology.

We fix a manifold $M$ endowed with a $\operatorname{SRF} \mathcal{F}$. We write $m=\operatorname{dim} M$ and $n=\operatorname{codim}_{M} \mathcal{F}$.

2.1. The BIC. A perversity is a map $\bar{p}: \mathrm{S}_{\mathcal{F}}^{\sigma} \rightarrow \overline{\mathbb{Z}}=\mathbb{Z} \cup\{-\infty, \infty\}$. There are several particular perversities:

- the constant perversity $\bar{c}$, defined by $\bar{c}(S)=c$, where $c \in \overline{\mathbb{Z}}$,

- the (basic) top perversity $\bar{t}$, defined by $\bar{t}(S)=n-\operatorname{codim}_{S} \mathcal{F}_{S}-2$, (cf. 1.2),

- the boundary perversity $\bar{\partial}$, defined by $\bar{\partial}=\min (\overline{0}, \bar{t})$.

The basic intersection cohomology (BIC for short) $\mathbb{H}_{\bar{p}}^{*}(M / \mathcal{F})$ is the cohomology of the complex $\Omega_{\bar{p}}^{*}(M / \mathcal{F})$ of $\bar{p}$-intersection basic forms. A $\bar{p}$-intersection basic form is a basic form defined on $R$ and having a vertical degree $\|\omega\|_{S}$, relative to a foliated tubular neighborhood $\left(T_{S}, \tau_{S}, S\right)$, lower than $\bar{p}(S)$ for each singular stratum $S$ (see $[18,19]$ for the exact definition). Recall that $\|\omega\|_{S} \leq i$ when $\omega\left(v_{0}, \ldots, v_{i},-\right)=0$ for each family $\left\{v_{0}, \ldots, v_{i}\right\}$ of vectors tangent to the fibers of $\tau_{S}$.

If depth $\mathrm{S}_{\mathcal{F}}=0$ then $\mathbb{H}_{\bar{p}}^{*}(M / \mathcal{F})=H^{*}(M / \mathcal{F})$ for any perversity $\bar{p}$.

2.2. Mayer-Vietoris. A covering $\{U, V\}$ of $M$ by saturated open subsets has a subordinate partition of unity made up of basic functions (see the

$\left({ }^{2}\right)$ For notions relating to basic cohomology we refer the reader to [5, 25], for basic intersection cohomology to $[18,19]$ and for intersection cohomology to $[6,2]$. 
lemma below). For such a covering we have the Mayer-Vietoris sequence

$$
0 \rightarrow \Omega_{\bar{p}}^{*}(M / \mathcal{F}) \rightarrow \Omega_{\bar{p}}^{*}(U / \mathcal{F}) \oplus \Omega_{\bar{p}}^{*}(V / \mathcal{F}) \rightarrow \Omega_{\bar{p}}^{*}((U \cap V) / \mathcal{F}) \rightarrow 0,
$$

where the maps are defined by restriction. The third map is onto since the elements of the partition of unity are $\overline{0}$-basic functions and $\Omega_{\overline{0}}^{*}(\bullet / \mathcal{F}) \cdot \Omega_{\bar{p}}^{*}(\bullet / \mathcal{F}) \subset$ $\Omega_{\bar{p}}^{*}(\bullet / \mathcal{F})$. Thus, the sequence is exact. This result is no longer true for more general coverings.

For the existence of the above Mayer-Vietoris sequence we need the following folklore result, well known for compact Lie group actions and regular riemannian foliations.

2.2.1. Lemma. A covering $\{U, V\}$ of $M$ by saturated open subsets has a subordinate partition of unity made up of basic functions.

2.3. Compact supports. In this note we need to work with the BIC with compact supports. The support of a differential form $\omega \in \Omega_{\bar{p}}^{*}(M / \mathcal{F})$, written $\operatorname{supp} \omega$, is the closure in $M$ of $\{x \in M \mid \omega(x) \neq 0\}$. We denote by $\Omega_{\bar{p}, \mathrm{c}}^{*}(M / \mathcal{F})$ the complex of $\bar{p}$-intersection basic forms with compact support. Its cohomology is $H_{\bar{p}, \mathrm{c}}^{*}(M / \mathcal{F})$. When $M$ is compact, we have $\mathbb{H}_{\bar{p}, \mathrm{c}}^{*}(M / \mathcal{F})=\mathbb{H}_{\bar{p}}^{*}(M / \mathcal{F})$, and if depth $\mathrm{S}_{\mathcal{F}}=0$ then $H_{\bar{p}, \mathrm{c}}^{*}(M / \mathcal{F})=H_{\mathrm{c}}^{*}(M / \mathcal{F})$ for any perversity $\bar{p}$.

Associated to a saturated open covering $\{U, V\}$ of $M$ we have the MayerVietoris sequence (see Lemma 2.2.1)

$$
\begin{aligned}
0 \rightarrow \Omega_{\bar{p}, \mathrm{c}}^{*}((U \cap V) / \mathcal{F}) \rightarrow \Omega_{\bar{p}, \mathrm{c}}^{*}(U / \mathcal{F}) \oplus \Omega_{\bar{p}, \mathrm{c}}^{*}(V / \mathcal{F}) & \rightarrow \Omega_{\bar{p}, \mathrm{c}}^{*}(M / \mathcal{F}) \rightarrow 0,
\end{aligned}
$$

where the maps are defined by inclusion. The third map is onto since the elements of the partition of unity are $\overline{0}$-basic functions. Thus, the sequence is exact.

2.4. ExAmple. Consider the isometric action $\Phi: \mathbb{R} \times \mathbb{S}^{2 d+2} \rightarrow \mathbb{S}^{2 d+2}$ given by the formula

$$
\Phi\left(t,\left(z_{0}, \ldots, z_{d}, x\right)\right)=\left(e^{a_{0} \pi i t} z_{0}, \ldots, e^{a_{d} \pi i t} z_{d}, x\right),
$$

with $\left(a_{0}, \ldots, a_{d}\right) \neq(0, \ldots, 0)$. Here, $\mathbb{S}^{2 d+2}=\left\{\left(z_{0}, \ldots, z_{d}, x\right) \in \mathbb{C}^{d} \times \mathbb{R} \mid\right.$ $\left.\left|z_{0}\right|^{2}+\cdots+\left|z_{d}\right|^{2}+x^{2}=1\right\}$. There are two singular strata: the north pole $S_{1}=(0, \ldots, 0,1)$ and the south pole $S_{2}=(0, \ldots,-1)$. The regular stratum is $\left.R=\mathbb{S}^{2 d+1} \times\right]-1,1[$. Let $r: R \rightarrow \mathbb{R}$ a smooth map, depending just on the $] 1,1[$ variable, with $r \equiv 0$ on $] 0,1 / 4[\cup] 3 / 4,1\left[\right.$ and $\int_{0}^{1} r=1$. The basic cohomology $H^{*}\left(\mathbb{S}^{2 d+2} / \mathcal{F}\right)$ of the foliation defined by this flow is the following:

\begin{tabular}{c|ccccccccc}
\hline$i$ & 0 & 1 & 2 & 3 & 4 & 5 & $\cdots$ & $2 d$ & $2 d+1$ \\
& 1 & 0 & 0 & {$[d r \wedge e]$} & 0 & {$\left[d r \wedge e^{2}\right]$} & $\cdots$ & 0 & {$\left[d r \wedge e^{d}\right]$} \\
\hline
\end{tabular}


where $e \in \Omega_{\frac{2}{2}}^{2}\left(\mathbb{S}^{2 d+2} / \mathcal{F}\right)$ is an Euler form (cf. [8]). These calculations come directly from the equalities

$$
\begin{aligned}
& \|r\|_{S_{k}}=0, \quad\|d r\|_{S_{k}}=-\infty, \quad\left\|e^{j}\right\|_{S_{k}}=\left\|r e^{j}\right\|_{S_{k}}=2^{j}, \\
& \left\|d r \wedge e^{j}\right\|_{S_{k}}=-\infty, \quad d r \wedge e^{j}=d\left(r e^{j}\right),
\end{aligned}
$$

for $k=1,2$ and $j \in\{1, \ldots, d\}$.

We notice that the top-dimensional basic cohomology group is isomorphic to $\mathbb{R}$, but this cohomology does not satisfy the Poincaré Duality in spite of the fact that the flow is isometric! The classical basic cohomology does not take into account the stratification $\mathbf{S}_{\mathcal{F}}$. However, even for the $\mathrm{SRF}$, the basic cohomology is finite-dimensional (cf. [25]).

If we consider the BIC of our example the picture changes. The following

\begin{tabular}{|c|c|c|c|c|c|c|c|c|c|c|c|}
\hline$i$ & 0 & 1 & 2 & 3 & 4 & 5 & 6 & 7 & $\cdots$ & $k-2$ & $k-1$ \\
\hline$\overline{<\overline{0}}$ & 0 & {$[d r]$} & 0 & {$[e \wedge d r]$} & 0 & {$\left[e^{2} \wedge d r\right]$} & 0 & {$\left[e^{3} \wedge d r\right]$} & $\ldots$ & 0 & {$\left[e^{d} \wedge d r\right]$} \\
\hline$\overline{0}, \overline{1}$ & 1 & 0 & 0 & {$[e \wedge d r]$} & 0 & {$\left[e^{2} \wedge d r\right]$} & 0 & {$\left[e^{2} \wedge d r\right]$} & $\ldots$ & 0 & {$\left[e^{d} \wedge d r\right]$} \\
\hline$\overline{2}, \overline{3}$ & 0 & 0 & {$[e]$} & 0 & 0 & {$\left[e^{2} \wedge d r\right]$} & 0 & {$\left[e^{3} \wedge d r\right]$} & $\cdots$ & 0 & {$\left[e^{d} \wedge d r\right]$} \\
\hline$\overline{4}, \overline{5}$ & 1 & 0 & {$[e]$} & 0 & {$\left[e^{2}\right]$} & 0 & 0 & {$\left[e^{3} \wedge d r\right]$} & $\ldots$ & 0 & {$\left[e^{d} \wedge d r\right]$} \\
\hline .. & $\ldots$ & . & $\cdots$ & $\ldots$ & .. & $\cdots$ & $\ldots$ & $\cdots$ & $\cdots$ & $\cdots$ & $\ldots$ \\
\hline$\overline{k-4}, \overline{k-3}$ & 1 & 0 & {$[e]$} & 0 & {$\left[e^{2}\right]$} & 0 & {$\left[e^{3}\right]$} & 0 & $\ldots$ & 0 & {$\left[e^{d} \wedge d r\right]$} \\
\hline$\geq \overline{k-2}$ & 1 & 0 & {$[e]$} & 0 & {$\left[e^{2}\right]$} & 0 & {$\left[e^{3}\right]$} & 0 & $\cdots$ & {$\left[e^{d}\right]$} & 0 \\
\hline
\end{tabular}
table presents the BIC $\mathbb{H}_{\bar{p}}^{*}\left(\mathbb{S}^{k=2 d+2} / \mathcal{F}\right)$ for the constant perversities:

These calculations come directly from the equalities (3).

We notice that the top-dimensional basic cohomology group is isomorphic either to 0 or $\mathbb{R}$. These cohomology groups are finite-dimensional. We recover the Poincaré Duality in the perverse sense:

$$
\mathbb{H}_{\bar{p}}^{*}\left(\mathbb{S}^{k} / \mathcal{F}\right) \cong \mathbb{H}_{\bar{q}}^{n-*}\left(\mathbb{S}^{k} / \mathcal{F}\right)
$$

for two complementary perversities: $\bar{p}+\bar{q}=\bar{t}=\overline{k-3}$.

This is more general. We have proved that the basic intersection cohomology is finite-dimensional and satisfies this perverse Poincaré Duality for the linear foliations (cf. [19, 20]) and for riemannian flows (cf. [16]).

3. Top class. The top group $H^{n}(M / \mathcal{F})$ of the basic cohomology of a regular riemannian foliation $\mathcal{F}$ defined on a connected compact manifold $M$ is $\mathbb{R}$ or 0 . Here $n=\operatorname{codim}_{M} \mathcal{F}$. We prove the same result for the top group $\mathbb{H}_{\bar{p}}(M / \mathcal{F})$ when $\mathcal{F}$ is a SRF defined on a connected compact manifold $M$. To do so, we need three lemmas. 
3.1. LemmA. Consider a foliated tubular neighborhood $\left(T_{S}, \tau_{S}, S\right)$ of a singular stratum $S \in \mathrm{S}_{\mathcal{F}}$. Fix a smooth function $\left.f:\right] 0,1[\rightarrow[0,1]$ with $f \equiv 0$ on ] $0,1 / 4]$ and $f \equiv 0$ on $[3 / 4,1[$. The map $[\omega] \mapsto[d f \wedge \omega]$ defines an isomorphism $\mathbb{I}_{\bar{p}, \mathrm{c}}^{*-1}\left(D_{S} / \mathcal{F}\right) \cong \mathbb{H}_{\bar{p}, \mathrm{c}}^{*}\left(D_{S} \times\right] 0,1[/ \mathcal{F} \times \mathcal{I})$, where $\mathcal{I}$ is the point foliation of the interval.

Proof. Consider the following differential complexes:

$$
\begin{array}{r}
\mathbb{A}^{*}=\left\{\omega \in \Omega_{\bar{p}}^{*}\left(D_{S} \times\right] 0,3 / 4[/ \mathcal{F} \times \mathcal{I}) \mid \operatorname{supp} \omega \subset K \times[c, 3 / 4[\right. \\
\text { for a compact } \left.K \subset D_{S} \text { and } 0<c<3 / 4\right\} \\
\left.\mathbb{B}^{*}=\left\{\omega \in \Omega_{\bar{p}}^{*}\left(D_{S} \times\right] 1 / 4,1[/ \mathcal{F} \times \mathcal{I}) \mid \operatorname{supp} \omega \subset K \times\right] 1 / 4, c\right] \\
\text { for a compact } \left.K \subset D_{S} \text { and } 1 / 4<c<1\right\} \\
\mathbb{C}^{*}=\left\{\omega \in \Omega_{\bar{p}}^{*}\left(D_{S} \times\right] 1 / 4,3 / 4[/ \mathcal{F} \times \mathcal{I}) \mid \operatorname{supp} \omega \subset K \times\right] 1 / 4,3 / 4[ \\
\text { for a compact } \left.K \subset D_{S}\right\} .
\end{array}
$$

Proceeding as in (2) we get the short exact sequence

$$
0 \rightarrow \Omega_{\bar{p}, \mathrm{c}}^{*}\left(D_{S} \times\right] 0,1[/ \mathcal{F} \times \mathcal{I}) \rightarrow \mathbb{A}^{*} \oplus \mathbb{B}^{*} \rightarrow \mathbb{C}^{*} \rightarrow 0 .
$$

The associated long exact sequence is

$$
\begin{aligned}
\cdots \rightarrow H^{i-1}\left(\mathbb{C}^{*}\right) \stackrel{\delta}{\rightarrow} \mathbb{H}_{\bar{p}, \mathrm{c}}^{i}\left(D_{S} \times\right] 0,1[/ \mathcal{F} \times \mathcal{I}) \rightarrow H^{i}\left(\mathbb{A}^{*}\right) \oplus H^{i}\left(\mathbb{B}^{*}\right) \\
\rightarrow H^{i}\left(\mathbb{C}^{*}\right) \rightarrow \cdots,
\end{aligned}
$$

where the connecting morphism is $\delta([\omega])=[d f \wedge \omega]$.

Before performing the calculation let us introduce some notation. Let $\beta$ be a differential form in $\Omega^{i}\left(D_{S} \times\right] a, b[)$ which does not include the $d t$ factor. Denote by $\int_{-}^{c} \beta(s) \wedge d s$ and $\int_{c}^{-} \beta(s) \wedge d s$ the forms in $\Omega^{i}\left(D_{S} \times\right] a, b[)$ obtained from $\beta$ by integration with respect to $s$, that is,

$$
\begin{aligned}
& \left(\int_{-}^{c} \beta(s) \wedge d s\right)(x, t)\left(\vec{v}_{1}, \ldots, \vec{v}_{i}\right)=\int_{t}^{c}\left(\beta(x, s)\left(\vec{v}_{1}, \ldots, \vec{v}_{i}\right)\right) d s \\
& \left(\int_{c}^{-} \beta(s) \wedge d s\right)(x, t)\left(\vec{v}_{1}, \ldots, \vec{v}_{i}\right)=\int_{c}^{t}\left(\beta(x, s)\left(\vec{v}_{1}, \ldots, \vec{v}_{i}\right)\right) d s
\end{aligned}
$$

where $c \in] a, b\left[,(x, t) \in D_{S} \times\right] a, b\left[\right.$ and $\left(\vec{v}_{1}, \ldots, \vec{v}_{i}\right) \in T_{(x, t)}\left(D_{S} \times\right] a, b[)$.

Each differential form $\omega \in \mathbb{A}^{*}, \mathbb{B}^{*}, \mathbb{C}^{*}$ can be written as $\omega=\alpha+d t \wedge \beta$ where $\alpha$ and $\beta$ do not contain $d t$. Consider a cycle $\omega=\alpha+d t \wedge \beta \in \mathbb{A}^{i}$ with supp $\omega \subset K \times\left[c, 3 / 4\left[\right.\right.$ for a compact $K \subset D_{S}$ and $0<c<3 / 4$. We have $\omega=-d\left(\int_{-}^{c / 2} \beta(s) \wedge d s\right)$. Since $\operatorname{supp} \int_{-}^{c / 2} \beta(s) \wedge d s \subset K \times[c, 3 / 4[$, we get $H^{*}\left(\mathbb{A}^{*}\right)=0$. In the same way, we get $H^{*}\left(\mathbb{B}^{*}\right)=0$. We conclude that

$$
\delta: H^{*-1}\left(\mathbb{C}^{*}\right) \rightarrow \mathbb{H}_{\bar{p}, \mathrm{c}}^{*}\left(D_{S} \times\right] 0,1[/ \mathcal{F} \times \mathcal{I})
$$

is an isomorphism. 
The foliated homotopy $\left.L: D_{S} \times\right] 1 / 4,3 / 4\left[\times[0,1] \rightarrow D_{S} \times\right] 1 / 4,3 / 4[$, defined by $L((u, t), s)=(u,(1-s) t+s / 2)$, satisfies $L(K \times] 1 / 4,3 / 4[\times[0,1]) \subset$ $K \times] 1 / 4,3 / 4\left[\right.$ for each compact $K \subset D_{S}$. So, the map $[\alpha] \mapsto[\alpha]$ induces an isomorphism

$$
I: H_{\bar{p}, \mathrm{c}}^{*}\left(D_{S} / \mathcal{F}\right) \rightarrow H^{*}\left(\mathbb{C}^{*}\right) .
$$

The isomorphism $\delta \circ I$ gives the result.

3.2. Lemma. Consider a foliated tubular neighborhood $T_{S}$ of a singular stratum $S \in \mathrm{S}_{\mathcal{F}}$. The inclusion $T_{S} \backslash S \hookrightarrow T_{S}$ induces an onto map

$$
\iota: \mathbb{H}_{\bar{p}, \mathrm{c}}^{n}\left(\left(T_{S} \backslash S\right) / \mathcal{F}\right) \rightarrow \mathbb{H}_{\bar{p}, \mathrm{c}}^{n}\left(T_{S} / \mathcal{F}\right) .
$$

Moreover, if $\bar{p}(S) \leq \bar{t}(S)$ then $\iota$ is an isomorphism.

Proof. We proceed in several steps.

(a) Rewriting $\mathbb{H}_{\bar{p}, \mathrm{c}}^{*}\left(T_{S} / \mathcal{F}\right)$. We have seen in [19] that we can identify $\Omega_{\bar{p}, \mathrm{c}}^{*}\left(T_{S} / \mathcal{F}\right)$ with the complex

$$
\begin{aligned}
\left\{\omega \in \Omega _ { \overline { p } , \mathrm { c } } ^ { * } \left(D_{S} \times\left[0,1[/ \mathcal{F} \times \mathcal{I})\left|\left\|\left.\omega\right|_{D_{S} \times\{0\}}\right\|_{\tau_{S}} \leq \bar{p}(S)\right. \text { and }\right.\right.\right. & \left.\left\|\left.d \omega\right|_{D_{S} \times\{0\}}\right\|_{\tau_{S}} \leq \bar{p}(S)\right\} .
\end{aligned}
$$

Here $\|-\|_{\tau_{S}}$ denotes the vertical degree relative to the fibration $\tau_{S}: D_{S} \equiv$ $D_{S} \times\{0\} \rightarrow S$. Recall that $\|0\|_{\tau_{S}}=-\infty$. Under this identification, the complex $\Omega_{\bar{p}, \mathrm{c}}^{*}\left(\left(T_{S} \backslash S\right) / \mathcal{F}\right)$ becomes $\Omega_{\bar{p}, \mathrm{c}}^{*}\left(D_{S} \times\right] 0,1[, \mathcal{F} \times \mathcal{I})$.

(b) Chasing $\mathbb{H}_{\bar{p}, \mathrm{c}}^{n}\left(T_{S} / \mathcal{F}\right)$. Consider the complex

$$
\mathbb{D}^{*}=\left\{\begin{array}{l|l}
\omega \in \Omega_{\bar{p}}^{*}\left(D_{S} \times[0,3 / 4[/ \mathcal{F} \times \mathcal{I})\right. & \begin{array}{l}
\left\|\left.\omega\right|_{D_{S} \times\{0\}}\right\|_{\tau_{S}} \leq \bar{p}(S), \\
\left\|\left.d \omega\right|_{D_{S} \times\{0\}}\right\|_{\tau_{S}} \leq \bar{p}(S) \text { and } \\
\operatorname{supp} \omega \subset K \times[0,3 / 4[ \\
\text { for a compact } K \subset D_{S}
\end{array}
\end{array}\right\}
$$

and the complexes $\mathbb{B}^{*}, \mathbb{C}^{*}$ as in the proof of Lemma 3.1. The short exact sequence

$$
0 \rightarrow \Omega_{\bar{p}, \mathrm{c}}^{*}\left(T_{S} / \mathcal{F}\right) \rightarrow \mathbb{D}^{*} \oplus \mathbb{B}^{*} \rightarrow \mathbb{C}^{*} \rightarrow 0
$$

produces the long exact sequence

$$
\begin{aligned}
\rightarrow H^{n-1}\left(\mathbb{D}^{*}\right) \oplus H^{n-1}\left(\mathbb{B}^{*}\right) \rightarrow H^{n-1}\left(\mathbb{C}^{*}\right) & \stackrel{\delta^{\prime}}{\rightarrow} \mathbb{H}_{\bar{p}, \mathrm{c}}^{n}\left(T_{S} / \mathcal{F}\right) \\
& \rightarrow H^{n}\left(\mathbb{D}^{*}\right) \oplus H^{n}\left(\mathbb{B}^{*}\right) \rightarrow H^{n}\left(\mathbb{C}^{*}\right) \rightarrow 0
\end{aligned}
$$

where the connecting morphism is $\delta^{\prime}([\omega])=[d f \wedge \omega]$ for a smooth map $f:[0,1[\rightarrow[0,1]$ with $f \equiv 0$ on $[0,1 / 4]$ and $f \equiv 0$ on $[3 / 4,1[$.

(c) Relating $H^{*}\left(\mathbb{C}^{*}\right)$ and $H^{*}\left(\mathbb{D}^{*}\right)$. We have already seen that $H^{*}\left(\mathbb{B}^{*}\right)=0$. Consider a cycle $\omega=\alpha+d t \wedge \beta \in \mathbb{D}^{n}$. For degree reasons, we have $\alpha(0)=0$ and then $\omega=d\left(\int_{0}^{-} \beta(s) \wedge d s\right)$. Since $\operatorname{supp} \int_{0}^{-} \beta(s) \wedge d s \subset K \times[0,3 / 4[$ we 
get $H^{n}\left(D^{*}\right)=0$. From the above long exact sequence, we obtain the exact sequence

$$
H^{n-1}\left(\mathbb{D}^{*}\right) \rightarrow H^{n-1}\left(\mathbb{C}^{*}\right) \stackrel{\delta^{\prime}}{\rightarrow} \mathbb{I}_{\bar{p}, \mathrm{c}}^{n}\left(T_{S} / \mathcal{F}\right) \rightarrow 0 .
$$

(d) Conclusion. Since the map $I$ is an isomorphism (cf. (4)) the composition $\delta^{\prime} \circ I: \mathbb{H}_{\bar{p}, \mathrm{c}}^{*-1}\left(D_{S} / \mathcal{F}\right) \rightarrow \mathbb{H}_{\bar{p}, \mathrm{c}}^{n}\left(T_{S} / \mathcal{F}\right)$ is an onto map. Lemma 3.1 shows that $\iota$ is an onto map. It remains to prove that $\iota$ is an isomorphism when $\bar{p}(S) \leq \bar{t}(S)$. We prove $H^{n-1}\left(\mathbb{D}^{*}\right)=0$. Proceeding as in (c) it suffices to consider a cycle $\omega=\alpha+d t \wedge \beta \in \mathbb{D}^{n-1}([0,3 / 4[)$ and show that $\alpha(0)=0$.

Suppose that $\alpha(0) \neq 0$. The foliation $\mathcal{F}$ induces a foliation $\mathcal{F}_{\tau_{S}}$ tangent to the fibers of $\tau_{S}: D_{S} \rightarrow S$ such that $\operatorname{dim} \mathcal{F}=\operatorname{dim} \mathcal{F}_{\tau_{S}}+\operatorname{dim} \mathcal{F}_{S}$ (cf. [13, 18]). By degree reasons, since $\alpha(0) \in \Omega_{\bar{p}, \mathrm{c}}^{n-1}\left(D_{S} / \mathcal{F}\right)$, we can write

$$
\begin{aligned}
\bar{t}(S) & \geq \bar{p}(S) \geq\|\alpha(0)\|_{\tau_{S}} \\
& =\underbrace{(\operatorname{dim} M-\operatorname{dim} S)-1}_{\text {dimension of the fiber of } \tau_{S}}-\underbrace{\left(\operatorname{dim} \mathcal{F}-\operatorname{dim} \mathcal{F}_{S}\right)}_{\operatorname{dim} \mathcal{F}_{\tau_{S}}}=\bar{t}(S)+1 .
\end{aligned}
$$

This contradiction gives $\alpha(0)=0$.

3.3. LEMMA. Let $N$ be a compact manifold endowed with a $R F \mathcal{N}$. If $O$ is a connected saturated open subset of $N$, then $H_{\mathrm{c}}^{n}(O / \mathcal{N})=0$ or $\mathbb{R}$.

Proof. We proceed in two steps.

The foliation $\mathcal{N}$ is transversally orientable. The result comes essentially from the basic Poincaré duality theorem for non-compact manifolds (cf. [21]). The foliation $\mathcal{N}_{O}$ is a transversally orientable complete foliation since $O$ is a saturated open subset of the compact manifold $N$. Then we have the isomorphism $H_{\mathrm{c}}^{n}(O / \mathcal{N}) \cong H^{0}(O / \mathcal{N}, \mathcal{P})$ where $\mathcal{P}$ is the homological orientation sheaf of $\mathcal{N}_{O}$. Since the manifold $O$ is connected, and the sheaf $\mathcal{P}$ is locally trivial and the stalk is $\mathbb{R}$, it follows that $H_{\mathrm{c}}^{n}(O / \mathcal{N})=0$ or $\mathbb{R}$.

General case. Consider the transverse orientation covering $\pi:\left(N^{*}, \mathcal{N}^{*}\right)$ $\rightarrow(N, \mathcal{N})$ (see [7]). The covering is given by a foliated action of $\mathbb{Z}_{2}$. The foliation $\mathcal{N}^{*}$ is a transversally orientable RF. We have the equality $H_{\mathrm{c}}^{n}(O / \mathcal{F})=$ $\left(H_{\mathrm{c}}^{n}\left(\pi^{-1}(O) / \mathcal{F}^{*}\right)\right)^{\mathbb{Z}_{2}}$. The subset $\pi^{-1}(O)$ is a saturated open subset of $N^{*}$. If $\pi^{-1}(O)$ is connected then the result comes from the previous case. Otherwise $\pi^{-1}(O)$ has two connected components foliated diffeomorphic to $O$ and the $\mathbb{Z}_{2}$-action interchanges them. The result now comes from the previous case.

The first result of this note is the following.

3.4. THEOREM. Let $M$ be a connected compact manifold endowed with a SRF $\mathcal{F}$. If $n=\operatorname{codim}_{M} \mathcal{F}$ and $\bar{p}$ is a perversity on $M$, then

$$
I_{\bar{p}}^{n}(M / \mathcal{F})=0 \text { or } \mathbb{R} .
$$


Proof. For each $i \in \mathbb{Z}$ we write:

- $\Sigma_{i} \subset M$ for the union of the strata of dimension less than or equal to $i$,

- $T_{i}$ for the tubular neighborhood of $\Sigma_{i}$ in $M \backslash \Sigma_{i-1}$.

We have $M \backslash \Sigma_{-1}=M$ and $M \backslash \Sigma_{m-1}=R$, where $m=\operatorname{dim} M$. Lemma 3.3 gives $H_{\mathrm{c}}^{n}(R / \mathcal{F})=0$ or $\mathbb{R}$. We get the result if we prove that the inclusion $M \backslash \Sigma_{i} \hookrightarrow M \backslash \Sigma_{i-1}$ induces an onto map

$$
H_{\mathrm{c}}^{n}\left(\left(M \backslash \Sigma_{i}\right) / \mathcal{F}\right) \rightarrow H_{\mathrm{c}}^{n}\left(\left(M \backslash \Sigma_{i-1}\right) / \mathcal{F}\right)
$$

for $i \in\{0, \ldots, m-1\}$.

From the open covering $\left\{M \backslash \Sigma_{i}, \bigcup_{\operatorname{dim} S=i} T_{S}\right\}$ of $M \backslash \Sigma_{i-1}$, we obtain the Mayer-Vietoris sequence

$$
\begin{aligned}
\bigoplus_{\operatorname{dim} S=i} H_{\mathrm{c}}^{n}\left(\left(T_{S} \backslash S\right) / \mathcal{F}\right) \rightarrow H_{\mathrm{c}}^{n}\left(\left(M \backslash \Sigma_{i}\right) / \mathcal{F}\right) \oplus \underset{\operatorname{dim} S=i}{\bigoplus_{\mathrm{c}}\left(T_{S} / \mathcal{F}\right)} & \rightarrow H_{\mathrm{c}}^{n}\left(\left(M \backslash \Sigma_{i-1}\right) / \mathcal{F}\right) \rightarrow 0 .
\end{aligned}
$$

Now, Lemma 3.2 gives the result.

Combining Theorem 3.4 with the tautness characterization of [17], we get

3.5. Corollary. Let $M$ be a connected compact manifold endowed with a SRF $\mathcal{F}$. Suppose that $\mathcal{F}$ is transversally orientable. Consider a perversity $\bar{p}$ on $M$ with $\bar{p} \leq \bar{t}$. If $n=\operatorname{codim}_{M} \mathcal{F}$, then the following two statements are equivalent:

(a) The foliation $\mathcal{F}_{R}$ is taut, where $R$ is the regular stratum of $(M, \mathcal{F})$.

(b) The cohomology group $\mathbb{H}_{\bar{p}}^{n}(M / \mathcal{F})$ is $\mathbb{R}$.

Proof. We know from [17] that (a) is equivalent to $H_{\mathrm{c}}^{n}(R / \mathcal{F})=\mathbb{R}$. So, it suffices to prove that $H_{\mathrm{c}}^{n}(R / \mathcal{F}) \cong H_{\bar{p}}^{n}(M / \mathcal{F})$ (cf. Lemma 3.3). We proceed as in the proof of the previous theorem changing "onto map" to "isomorphism".

3.6. Remarks. (a) The perversity $\bar{p}=\overline{-\infty}$ satisfies $\bar{p} \leq \bar{t}$. In this case the group $\mathbb{H}_{\bar{p}}^{n}(M / \mathcal{F})$ becomes $H^{n}(M / \mathcal{F}, \Sigma / \mathcal{F})$. Here, the relative basic cohomology $\mathbb{H}_{\bar{p}}^{*}(M / \mathcal{F}, \Sigma / \mathcal{F})$ is computed from the relative basic complex

$$
\Omega^{*}(M / \mathcal{F}, \Sigma / \mathcal{F})=\left\{\omega \in \Omega^{*}(M / \mathcal{F}) \mid \omega \equiv 0 \text { on } \Sigma\right\} .
$$

(b) The boundary perversity $\bar{\partial}$ satisfies $\bar{\partial} \leq \bar{t}$. In this case the group $\mathbb{I} H_{\bar{\partial}}^{n}(M / \mathcal{F})$ becomes $H^{n}(M / \mathcal{F}, \partial(M / \mathcal{F}))$. Here, the relative basic cohomology $H^{*}(M / \mathcal{F}, \partial(M / \mathcal{F}))$ is computed from the relative basic complex $\Omega^{*}(M / \mathcal{F}, \partial(M / \mathcal{F}))=\left\{\omega \in \Omega^{*}(M / \mathcal{F}) \mid \omega \equiv 0\right.$ on the boundary strata $\}$. In particular, when the boundary strata do not appear then $\mathbb{H}_{\bar{\partial}}(M / \mathcal{F})=$ $H^{n}(M / \mathcal{F})$. 
(c) If $\bar{p} \not \subset \bar{t}$ then the group $\mathbb{H}_{\bar{p}}^{n}(M / \mathcal{F})$ does not establish the tautness of $(R, \mathcal{F})$. For example, we always have $\mathbb{H}_{\bar{t}+\overline{1}}^{n}(M / \mathcal{F})=H^{n}((M \backslash \Sigma) / \mathcal{F})=0$ if $\Sigma \neq \emptyset$.

(d) In Theorem 3.4 and Corollary 3.5 we can suppose that the manifold $M$ is not compact and replace $\mathbb{H}_{\bar{p}}^{n}(M / \mathcal{F})$ by $\mathbb{H}_{\bar{p}, \mathrm{c}}^{n}(M / \mathcal{F})$.

\section{References}

[1] H. Boualem and P. Molino, Modèles locaux saturés de feuilletages riemanniens singuliers, C. R. Acad. Sci. Paris 316 (1993), 913-916.

[2] J.-L. Brylinski, Equivariant intersection cohomology, in: Contemp. Math. 139, Amer. Math. Soc., 1992, 5-32.

[3] D. Domínguez, Finiteness and tenseness theorems for riemannian foliations, Amer. J. Math. 120 (1998), 1237-1276.

[4] A. El Kacimi et G. Hector, Décomposition de Hodge basique pour un feuilletage riemannien, Ann. Inst. Fourier (Grenoble) 36 (1986), no. 3, 207-227.

[5] A. El Kacimi, V. Sergiescu and G. Hector, La cohomologie basique d'un feuilletage riemannien est de dimension finie, Math. Z. 188 (1985), 593-599.

[6] M. Goresky and R. MacPherson, Intersection homology theory, Topology 19 (1980), $135-162$.

[7] G. Hector and U. Hirsch, Introduction to the Geometry of Foliations. Part A, Aspects Math., Vieweg, 1981.

[8] G. Hector and M. Saralegi-Aranguren, Intersection cohomology of $\mathbb{S}^{1}$-actions, Trans. Amer. Math. Soc. 338 (1993), 263-288.

[9] F. W. Kamber et Ph. Tondeur, Harmonic Foliations, Lecture Notes in Math. 949, Springer, 1982, 87-121.

[10] X. Masa, Duality and minimality in riemannian foliations, Comment. Math. Helv. 67 (1992), 17-27.

[11] V. Miguel and R. Wolak, Minimal singular riemannian foliations, C. R. Math. Acad. Sci. Paris 342 (2006), 33-36.

[12] P. Molino, Feuilletages riemanniens réguliers et singuliers, in: Géométrie différentielle (Paris, 1986), Hermann, Paris, 1988, 173-201.

[13] —, Riemannian Foliations, Progr. Math. 73, Birkhäuser, 1988.

[14] —, Orbit-like foliations, in: Geometric Study of Foliations (Tokyo, 1993), World Sci., Singapore, 1994, 97-119,

[15] P. Molino et V. Sergiescu, Deux remarques sur les flots riemanniens, Manuscripta Math. 51 (1985), 145-161.

[16] J. I. Royo Prieto, Estudio cohomológico de flujos riemannianos, Ph.D. Universidad del País Vasco/Euskal Herriko Unibertsitatea, 2003, http://www.ehu.es/joseroyo/pdf/tesis.pdf.

[17] J. I. Royo Prieto, M. Saralegi-Aranguren and R. Wolak, Tautness for riemannian foliations on non-compact manifolds, ArXiv.mathDG/0505675.

[18] M. Saralegi-Aranguren and R. Wolak, The BIC of a conical fibration, Math. Notes 77 (2005), 213-231.

[19] - - - The BIC of a defined by an abelian group of isometries, ArXiv.mathDG/0401407.

[20] - - - The Poincaré duality of a Killing foliation, preprint. 
[21] V. Sergiescu, Cohomologie basique et dualité des feuilletages riemanniens, Ann. Inst. Fourier (Grenoble) 35 (1985), no. 3, 137-158.

[22] P. Stefan, Accessible sets, orbits, and foliations with singularities, Proc. London Math. Soc. 29 (1974), 699-713.

[23] H. J. Sussmann, Orbits of families of vector fields and integrability of distributions, Trans. Amer. Math. Soc. 180 (1973), 171-188.

[24] Ph. Tondeur, Geometry of Foliations, Monogr. Math. 90, Birkhäuser, 1997.

[25] R. Wolak, Basic cohomology for singular riemanian foliations, Monatsh. Math. 128 (1999), 159-163.

José Ignacio Royo Prieto

Departamento de Matemática Aplicada

Universidad del País Vasco

Alameda de Urquijo s/n, 48013 Bilbao, Spain

E-mail: joseignacio.royo@ehu.es

Robert Wolak

Institute of Mathematics

Jagiellonian University

Reymonta 4

30-059 Kraków, Poland

E-mail: robert.wolak@im.uj.edu.pl
Martintxo Saralegi-Aranguren

Fédération CNRS

Nord-Pas-de-Calais FR 2956

UPRES-EA 2462 LML

Faculté Jean Perrin

Université d'Artois

Rue Jean Souvraz SP 18

62307 Lens Cedex, France

E-mail: saralegi@euler.univ-artois.fr

Received June 6, 2005;

received in final form January 4, 2006 